\title{
Satzarten unterscheiden - Kann das der Computer? Syntaktische Explorationen anhand von COSMAS II
}

\author{
Anton Näf (Neuchâtel)
}

\begin{abstract}
Is the computer capable of recognizing different sentence types in a linguistic corpus such as COSMAS II (Mannheim), which has not been previously treated by a tagger or a parser? The answer is in fact no. However, in the present article it is shown that under certain circumstances an automatic distinction is nevertheless possible. Making use of a procedure that we have called Anfragezuspitzung (literally: making a query pointed; encirclement of a grammatical phenomenon by a combination of several specific queries), and taking as a starting point philological prior knowledge that has been gathered "by hand", it proves to be perfectly possible to arrive at a satisfactory result. With the example of sentence types in German, in particular the distinction between interrogative and exclamatory sentences, we demonstrate in this article that such a distinction can be carried out automatically with a high degree of accuracy, e.g. the distinction between War das eine gute Idee? (Was this a good idea?) and War das eine gute Idee! (What a good idea this was!).
\end{abstract}

\section{$1 \quad$ Einleitung}

Dass die am IDS in Mannheim installierte Corpus-Datenbank COSMAS II (Corpus Search, Management and Analysis System) sich für Forschungen im Bereich der Lexikologie - sowie deren angewandter Schwesterdisziplin, der Lexikographie - als von grossem Nutzen erweist, ist unmittelbar einsichtig und unterdessen auch allgemein bekannt und anerkannt. Eines ist klar: Wer heutzutage ein Wörterbuch verfasst oder auch bloss aktualisiert, kommt um die Benutzung digitaler Textdatenbanken nicht mehr herum. Das Bestreben jedes Wörterbuchverfassers sollte es ja sein, ein möglichst getreues Abbild von Vorkommen und Gebrauch der Lexeme einer Sprache zu bieten. Bei der Abfassung eines Wörterbuchartikels steht dem Lexikographen nun dank den digitalen Grosskorpora sozusagen in Sekundenschnelle ein Belegmaterial mit - zum Teil allerdings bloss impliziten - Informationen bezüglich Gebrauch, Kookkurrenzen und Unterbedeutungen der zur Debatte stehenden Lexeme zur Verfügung, von dem er noch vor wenigen Jahren bloss hätte träumen können. Auch für die Wortbildung des Deutschen eröffnen sich dank diesem neuen Instrumentarium völlig neue Perspektiven, etwa bei der empiriegeleiteten Unterscheidung von okkasionellen und usuellen Komposita (beispielsweise mit Sport- als Vorderglied bzw. -sport als Hinterglied); von diesen haben im allgemeinen nur die letzteren - zumindest in Auswahl - ihren Platz in einem Wör- 
terbuch. Eines scheint klar: ein künftiges, nach einer tabula rasa aus den Quellen neu erarbeitetes Wörterbuch der deutschen Gegenwartssprache dürfte sich von den heute im Umlauf befindlichen ziemlich stark unterscheiden, sowohl bezüglich Bestand und Gewichtung der ausgewählten Lexeme als auch - und wohl vor allem - bezüglich Auswahl und Reihenfolge der berücksichtigten Unterbedeutungen.

\section{Syntaxforschung mit COSMAS II}

COSMAS II, mit einem Gesamtumfang von über einer Milliarde öffentlich zugänglichen Textwörtern das grösste - und darüber hinaus kostenlos fernabfragbare - Korpus zur deutschen Gegenwartssprache, ist nun allerdings weder mit einem Tagger (Hinzufügung der Wortklassen) noch mit einem Parser (Markierung der syntaktischen Strukturen) "vorbehandelt". Es scheint also im Prinzip für grammatische Fragestellungen zunächst einmal wenig geeignet. Da im Abfrage- und Analyseprogramm von COSMAS II keinerlei linguistische Markierungen vorgesehen sind, kann man mit diesem Forschungsinstrument natürlich auch keine entsprechenden Suchanfragen stellen, z.B. solche nach Wortklassen (Verb, Adjektiv, Präposition, usw.) oder deren grammatischen Unterkategorien (Konjunktiv II, Dativ, Superlativ, usw.) oder gar nach syntaktischen Funktionen (Subjekt, Präpositionalattribut, Apposition, usw.).

Dass COSMAS II nicht grammatisch annotiert ist, bedeutet nun aber nicht, dass es für grammatische Fragestellungen unbrauchbar wäre. All die gar nicht so seltenen grammatischen Phänomene, die in irgendeiner Art an konkrete Wörter gebunden sind, etwa an die - geschlossenen - Klassen der Funktionswörter (Präpositionen, Konjunktionen, Modalpartikeln, u.a.), können mit Hilfe von COSMAS II zumindest indirekt, d. h. über den "Umweg" über einzelne Lexeme, angegangen werden. ${ }^{1}$ Hingegen ist COSMAS II natürlich blind für die Erfassung von "reinen Strukturen". Man kann also damit in einem vordefinierten Korpus beispielsweise nicht nach allen Ketten wie in (1) suchen:

(1) (a) $\{$ Det+Adj+Nomen $\}$

(b) $\left\{\right.$ Det+Nomen $\left.+[\text { Det+Nomen }]^{\mathrm{Gen}}\right\}$

(Typ das unwegsame Gelände)

(Typ der Sinn des Lebens)

A fortiori ist COSMAS II nicht imstande, semantisch abgegrenzte Klassen von syntaktischen Phänomenen aus einem Korpus herauszudestillieren, z.B. im Bereich der Nebensatz-Syntax alle Konditionalsätze. Die Schwierigkeiten mit einem solchen Begriff liegen in erster Linie darin, dass etwa die - bezüglich ihrer "Oberflächenstruktur" so unterschiedlichen - Konstruktionen in (2) beide eine Voraussetzung (hier genauer: Eventualität) ausdrücken, was bislang noch jenseits des "Verstehenshorizonts" von automatischen Analyseprogrammen bleibt.

(2) (a) Wenn das Geld dafür nicht reicht, ...

(b) Sollte das Geld dafür nicht reichen, ...

\footnotetext{
${ }^{1}$ Lemnitzer/Zinsmeister (2006: 136) sprechen in diesem Zusammenhang von "wortbasierter Suche".
} 


\section{Einkreisung von syntaktischen Phänomenen durch Anfragezuspitzung}

Man könnte nun hier abbrechen und resignierend feststellen, dass eben mit einem AnalyseTool wie COSMAS II in der Syntax kein Staat zu machen sei und dass damit einem Phänomen wie beispielsweise den Konditionalsätzen nicht beizukommen sei. Das wäre aber eine viel zu pessimistische Sicht der Dinge. Wenn man unter Aktivierung von linguistischem Vorwissen und mit Hilfe des Verfahrens der Anfragezuspitzung ${ }^{2}$ dem Suchwerkzeug etwas "auf die Sprünge hilft", lassen sich auch komplexe Phänomene wie der Konditionalsatz insgesamt doch recht gut "einkreisen". Es gilt dabei, bei der Suche zunächst einmal von geeigneten Wortkörpern auszugehen. Das prototypische Ausdrucksmittel für Bedingungen ist die Konjunktion wenn. Deshalb wird man sich hier zunächst durch einen ersten Suchbefehl alle wenn-Sätze eines gegebenen Korpus zusammenstellen lassen. Unter diesen sind aber ein beträchtlicher Teil keine Konditionalsätze wie in (2a), sondern unter anderem Temporalsätze, mit unterschiedlichen semantischen Werten wie z.B. in (3a) und (3b).

(3) (a) [Ich glaube nicht an den Ruhestand.] Wenn Sie mit Arbeiten aufhören, dann ist es fertig. $(\rightarrow$ sobald/*immer wenn)

(b) Wenn ich morgens aufstehe, ist es noch dunkel. ( $\rightarrow$ immer wenn/*sobald)

Die Konjunktion wenn tritt im COSMAS-Teilkorpus Zürcher Tagesanzeiger (Jahrgänge 1996-2000, total 61 Mio. Textwörter, im Folgenden als "zta" zitiert) insgesamt 100'954 mal auf. Natürlich wäre es sehr angenehm, wenn ein automatisches Analyseprogramm imstande wäre, die wenn-Sätze den einzelnen semantischen Unterkategorien zuzuordnen; aber davon sind wir noch weit entfernt. Eine Handauswertung einer Zufallsauswahl von 200 Belegen $^{3}$ aus dem zta-Korpus ergibt, dass es sich bei knapp der Hälfte davon um Konditionalsätze und bei einem Viertel um Temporalsätze handelt. Aber während man sich noch vorstellen kann, dass sich durch eine immer genauere Nachahmung der Funktionsweise des menschlichen Gehirns derartige Unterscheidungen in Zukunft einmal programmieren lassen, stösst man wohl dort auf prinzipiell unüberwindbare Grenzen, wo auch die philologische "Handarbeit", trotz Berücksichtigung von Sprechsituation und Kotext, zu keinem eindeutigen Resultat gelangt. Gar nicht so selten sind nämlich Fälle wie (4), bei denen sowohl eine konditionale ( $\rightarrow$ falls) als auch eine temporale ( $\rightarrow$ immer wenn/sobald) Interpretation einen befriedigenden, wenn auch leicht verschiedenen Sinn ergibt.

(4) Wenn man gewinnt, finden einen im nachhinein viele gut. (zta 10.01.96)

Andere lexemgebundene Ausdrucksmöglichkeiten für das Bedingungsverhältnis, z.B. die Subjunktion falls oder Einleitegruppen wie unter der Voraussetzung dass oder gesetzt den

\footnotetext{
${ }^{2} \mathrm{Zu}$ diesem Begriff vgl. Duffner/Näf in diesem Band.

${ }^{3}$ Die Handauswertung von 200 Belegen nimmt etwa drei Stunden Zeit in Anspruch, dies auch deswegen, weil die Darstellung als KWIC-Konkordanz in vielen Fällen für eine zweifelsfreie Zuordnung nicht genügt. In diesen Fällen muss das gesamte Satzgefüge oder gar der ganze Textabschnitt als Kontext herbeigeklickt werden. Auch bei fortschreitender Automatisierung werden also die Linguisten (oder vielleicht sollte man besser sagen: die Philologen) keinesfalls überflüssig. Der entscheidende Vorteil gegenüber "früher" ist aber, dass sie ihre Zeit heute statt der Belegsuche der - geistig viel anspruchsvolleren - Beleginterpretation widmen können.
} 
Fall (dass) lassen sich durch entsprechende Suchbefehle ebenfalls schnell auffinden. Da diese offenbar nicht polysem sind, braucht es hier keine nachträglichen Sortierungsprozeduren.

Als viel schwieriger erweist sich hingegen die Erfassung der Konditionalsätze mit VerbErststellung vom Typ (2b). Da COSMAS II weder "Verb" noch "Erststellung" versteht, ist man hier mit der automatischen Erfassung von Belegen an sich am Ende angelangt. Immerhin kann man auch hier durch die Mobilisierung von grammatischem Vorwissen ein Stück weiterkommen. Falls der intuitive Eindruck zutrifft, dass bei diesem Satztyp als Verben typischerweise Auxiliarverben ${ }^{4}$ und Modalverben auftreten, und zwar vorzugsweise im Konjunktiv II, wäre damit ein erster "Hebel" gegeben, um zu möglichst repräsentativen Daten zu kommen: hätte(n), wäre(n), sollte(n), könnte(n),wollte(n), müsste(n), dürfte(n). ${ }^{5}$ Wenn man darüber hinaus noch unterstellt, dass Verb-Erst-Konditionalsätze stellungsmässig vor allem als Vordersätze auftreten, so kann noch gezielter nach allen entsprechenden Verbformen am Satzanfang (genauer: Satzgefüge-Anfang) gesucht werden. Und da erweist sich nun als sehr wertvoll, dass COSMAS II dank der Einstellungsoption "Grossschreibung berücksichtigen" zwischen Minuskel- und Majuskelbelegen unterscheiden kann. Hier eine Verteilungstabelle zum zta-Korpus:

\begin{tabular}{|l|r|r|r|r|r|r|r|}
\hline Lexem & HÄTTE(N) & WÄRE(N) & KÖNNTE(N) & SOLLTE(N) & WOLLTE(N) & DÜRFTE(N) & MÜSSTE(N) \\
\hline $\begin{array}{l}\text { Total } \\
\text { Belege }\end{array}$ & $48^{\prime} 117$ & $37^{\prime} 881$ & $39^{\prime} 163$ & $29 ' 789$ & $25^{\prime} 006$ & $15^{\prime} 395$ & $15^{\prime} 345$ \\
\hline $\begin{array}{l}\text { Davon } \\
\text { Majus- } \\
\text { kel- } \\
\text { Belege }\end{array}$ & $3.1 \%$ & $4.3 \%$ & $1.3 \%$ & $\mathbf{1 0 . 8 \%}$ & $0.9 \%$ & $0.1 \%$ & $1.6 \%$ \\
\hline
\end{tabular}

Tab. 1: Total der Belege und Anteil der Majuskelbelege im zta-Korpus (Zürcher Tages-Anzeiger 1996-2000)

Was in dieser Tabelle auffällt, ist eine klare Distributionspräferenz, ${ }^{6}$ nämlich der im Vergleich zu den übrigen Modalverben hohe Anteil an Majuskelbelegen bei sollen. Nun könnte man denken, dieser komme aufgrund der Homonymie mit den Präteritum-Formen zustande. Dies ist aber nicht der Fall. Bei einer Zufallsauswahl von 200 Belegen aus dem zta-Korpus liegt in allen Sollte-Sätzen der Konjunktiv II vor. Was die Satzart betrifft, handelt es sich dabei bei $93 \%$ um Konditionalsätze wie etwa (5a) und bloss bei $7 \%$ um Verb-Erst-Interrogativsätze wie etwa $(5 b)$

(5) (a) Sollte ich es aber nicht schaffen, ist nichts verloren. (zta 16.03.96)

(b) Sollte man der gleichgültigen Mehrheit das Stimmrecht entziehen? (zta 26.01.96)

\footnotetext{
${ }^{4} \mathrm{Vgl}$. auch so bekannte Zitate wie Und bist du nicht willig, so brauch ich Gewalt (Goethe) oder Bist du bei mir, geh ich mit Freuden zum Sterben und zu meiner Ruh (Bach).

${ }^{5}$ Bei sollen und wollen ist die Sachlage insofern komplizierter, als deren Präteritum- und Konjunktiv-II-Formen homonym sind. Nicht berücksichtigt in Tab. 1 ist das Modalverb möchten, das man meines Erachtens synchronisch besser als eigenes Verb (und nicht als Konjunktiv II von mögen) ansehen sollte.

${ }^{6}$ Vgl. zu diesem Begriff Heid (2005: 106).
} 
Diese Beobachtungen führen uns nun zum Schluss, dass sich die Konstruktion "Sollte $(n)+$ Infinitiv" im heutigen Deutsch in hohem Masse auf den Ausdruck von Bedingungsverhältnissen (Eventualität) spezialisiert hat - als Konkurrenzstruktur zu den wenn-Sätzen. Sollte(n) tritt in konditionalen Vordersätzen nicht nur viel häufiger auf als die andern Modalverben, sondern auch häufiger als die Auxiliarverben Wäre(n) und Hätte(n) (vgl. Tab. 1). Dieses von den Grammatiken und den DaF-Lehrwerken bislang noch weitgehend unbemerkte sprachliche Phänomen ${ }^{7}$ konnte hier zwar noch nicht vollständig etabliert, aber doch schon in den Konturen "eingekreist" werden. Eine Kookkurrenzanalyse aller einschlägigen Belege erlaubt darüber hinaus, für diesen syntaktischen Rahmen - sozusagen auf Knopfdruck - die prototypischen lexikalischen Füllungen zu extrahieren, z.B. Serien wie Sollte es zutreffen/sich herausstellen/sich bewahrheiten, dass ... oder Sollte x scheitern/gelingen/gut gehen ... Derartige für die Sprachwirklichkeit repräsentative "Satzbausteine" sind für Deutschlernende viel nützlicher als die nur allzu oft verquer geratenen Beispielsätze der Grammatiken und DaFLehrbücher.

Vorläufiges Fazit: auch wenn man mit dem Analyseinstrument COSMAS II ein komplexes syntaktisch-semantisches Phänomen wie etwa die Konditionalsätze im Deutschen nicht direkt untersuchen kann, so lassen sich doch durch allerlei Um- und Schleichwege mindestens relevante Teilmengen davon anpeilen. Das bedeutet: Durch eine Kombination von verschiedenen Einzelanfragen ("Anfragezuspitzung") kann man mit COSMAS II zahlreiche syntaktische Phänomene zwar nicht wirklich exhaustiv erfassen, aber doch zumindest in ihren Konturen "einkreisen". Jedenfalls darf man behaupten, dass man im Vergleich zur "vordigitalen" Phase der Linguistik auch in der Syntax - bei einem vertretbaren Aufwand an Zeit und Energie - zu durchaus relevanten Forschungsresultaten gelangen kann, dies durch eine Verbindung von automatischer Korpusanalyse und philologischer Handarbeit.

\section{$4 \quad$ Anwendung auf die Unterscheidung von Satzarten}

Im Folgenden soll anhand von zwei für die Forschungsgeschichte zentralen Beispielen gezeigt werden, dass das Abfragetool von COSMAS II sich auch mit Gewinn zur Klärung offener Fragen im Bereich der Satzarten (im Sinne von Hauptsatzarten) einsetzen lässt. Was die Frage der für das Deutsche anzusetzenden Typen von Satzarten oder Satzmodi, insbesondere auch was die Kategorie Exklamativsatz betrifft, muss hier auf die einschlägige Spezialliteratur verwiesen werden. ${ }^{8}$ Die gängigen Definitionen der Satzarten rekurrieren sowohl auf lexemgebundene als auch auf grammatische (morphologische und syntaktische) Kriterien. Es ist klar, dass in einem syntaktisch nicht annotierten Korpus vor allem die ersteren als "Hebel" dienen müssen, in erster Linie die (aufzählbaren) w-Wörter. Prinzipiell nichts anfangen dagegen kann COSMAS II mit den für die Satzartenunterscheidung zentralen morphologischen (Verbmodus) und syntaktischen (Verbstellung) Definitionskriterien. Das Gleiche gilt für die bei einem schriftkonservierten Korpus ja nicht verfügbare Intonation.

\footnotetext{
7 Allerdings spricht nun die neueste Auflage der Duden-Grammatik ( ${ }^{7} 2005$ : 566) von sollte "als eine[r] Art Hilfsverb des Potenzialis in Bedingungssätzen und rhetorischen Fragen".

${ }^{8}$ Vgl. dazu Altmann (1993), Näf (1995) und (1996).
} 
Aber auch hier ist deswegen noch nicht alles verloren: Da das COSMAS-Korpus den Zugriff auf die Satzschlusszeichen erlaubt, - und diese sind ja nichts anderes als ein, wenn auch bloss unvollkommenes, Abbild der Intonation -, wird über diesen "Umweg" die automatische Extraktion von - im Hinblick auf eine anschliessende Handanalyse - relevantem Sprachmaterial möglich. Insgesamt ergeben sich damit auch für die Frage der Satzarten - trotz der auf den ersten Blick aussichtslosen Ausgangslage - interessante Zugriffsmöglichkeiten. Wie man durch eine Verbindung von "Anfragezuspitzung" und Einspeisung von einschlägigem - durch Auswertung nicht digitaler Korpora erworbenem - grammatischem Vorwissen auch hier zu beachtlichen Ergebnissen kommen kann, soll nun exemplarisch aufgezeigt werden, zunächst an einer Teilmenge von Verb-Erst-Sätzen und anschliessend einer solchen von Verb-ZweitSätzen. Das Hauptaugenmerk soll dabei auf die Abgrenzung der Interrogativsätze von den strukturähnlichen Exklamativsätzen gelegt werden.

\subsection{Verb-Erst-Sätze}

Die fünf im heutigen Deutsch wichtigsten Satzstrukturen mit finitem Verb in Erststellung sind die folgenden (die Reihenfolge in Tabelle 2 ist die ihrer vermuteten Auftretensfrequenz): ${ }^{9}$

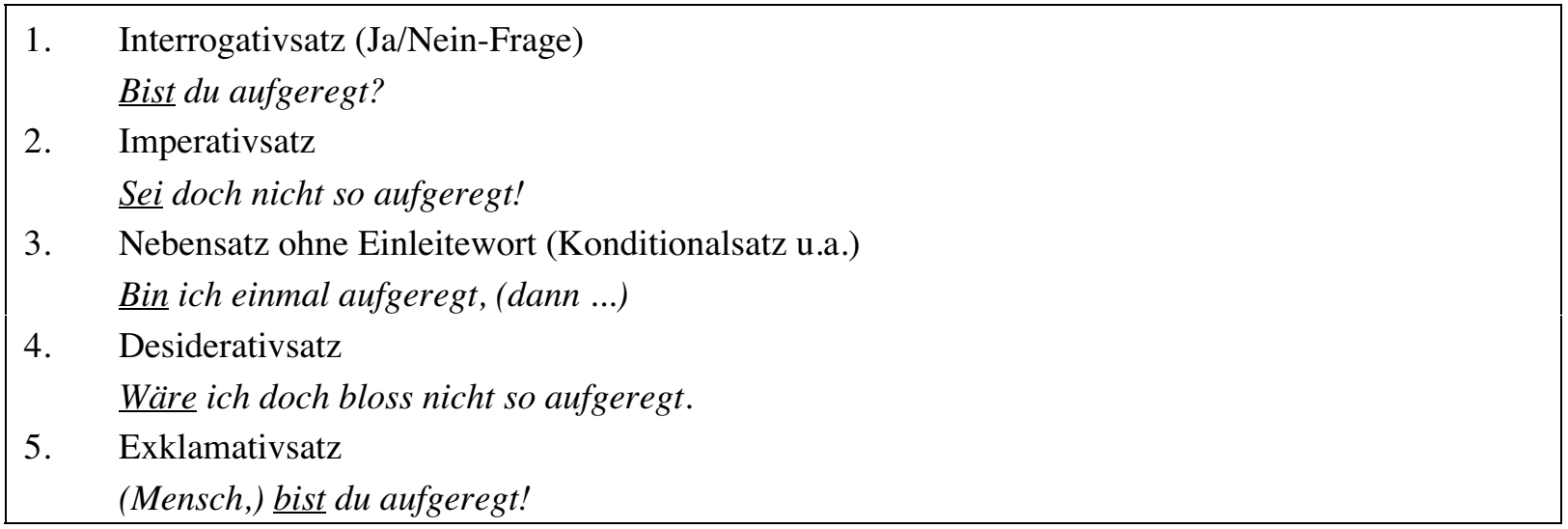

Tab. 2: Verb-Erst-Strukturen im Gegenwartsdeutsch

Im Folgenden interessieren wir uns primär für die Möglichkeiten einer semi-automatischen Unterscheidung der obigen Typen 1 und 5, die in den gleichen Modi (Indikativ, Konjunktiv II) auftreten können. Der Typ 2 (Imperativsatz) steht insofern etwas abseits, als für ihn als einzigen der Verbmodus Imperativ konstitutiv ist. Da COSMAS II jedoch die Kategorie Imperativ nicht erkennen kann, ist dieser - für den menschlichen Betrachter leicht diagnostizierbare - Typ mit diesem Tool nicht extrahierbar.

Für die Unterscheidung der Satzarten haben sich intonatorische Minimalpaare als das "ökonomischste" Testmittel herausgestellt. Ausgangspunkt unserer Überlegungen soll deshalb das (ad hoc konstruierte) intonatorische Minimalquadrupel (!) in Tabelle 3 sein. Wie durch eine Transformation des Satzes SCHLAFEN SIE GUT von der Höflichkeitsform in die Du-Form unmittelbar einsichtig wird, kann diese mehrdeutige (homographe) Struktur je nach Intonati-

\footnotetext{
${ }^{9}$ Duden-Grammatik $\left({ }^{5} 1995: 785\right)$ und $\left({ }^{7} 2005: 876\right)$.
} 
on, Hauptakzent, Verträglichkeit mit Modalpartikeln u.a. auf die vier folgenden Weisen interpretiert werden.

\section{\{SCHLAFEN SIE GUT $\}$}

(a) Schläfst du GUT?

(b) SCHLAF gut!

(c) Schläfst DU (aber) gut!

(d) Schläfst du gut, (dann ...)
Est-ce que tu dors bien?/Do you sleep well?

Dors bien./Sleep well!

Comme tu dors bien!/How well you sleep!

Si tu dors bien .../If you sleep well ...

Tab. 3: Disambiguierung intonatorischer Verb-Erst-Minimalpaare

Besehen wir uns nun von der Nähe, wodurch sich die - insgesamt viel frequentere - Interrogativsatzstruktur (a) von der Exklamativsatzstruktur (c) trotz aller Ähnlichkeiten im einzelnen unterscheidet. Zuerst gilt es festzuhalten, dass ein Exklamativsatz nur dann möglich ist, wenn der Prädikatsausdruck des Satzes (Verb, Adjektiv) in irgendeiner Art (quantitativ oder qualitativ) graduierbar ist. ${ }^{10}$ Von dieser semantischen Restriktion abgesehen kann aber bei beiden Satztypen im Prinzip jedes Verb auftreten, vgl. Minimalpaare wie die folgenden:
(a) Bist du MÜde?
vs. Bist DU müde!
(b) Hast du HUNGer?
vs. Hast DU Hunger!
(c) Kommt ihr SPÄT nach Hause?
vs. Kommt IHR spät nach Hause!
(d) Bekommen Sie VIEle Mails?
vs. Bekommen SIE viele Mails!

Obwohl vom Sprachsystem her somit offenbar keinerlei diesbezügliche Restriktionen bestehen, scheint es nun aber in der Sprachwirklichkeit doch so zu sein, dass bei den Verb-ErstExklamativsätzen - im Gegensatz zu den entsprechenden Interrogativsätzen - de facto fast nur die beiden Auxiliarverben sein und haben auftreten (Näf 1996 147f.)! Aber das ist noch nicht alles: In den prototypischen Exklamativsatz-Belegen folgt auf das Verb wie in (6) ein offenbar stets den Hauptakzent tragendes - Personalpronomen bzw. (in der dritten Person Sing. und Plural) ein der-Demonstrativpronomen, ${ }^{11}$ meist in der Rolle des Subjektspronomens.

Ausgerüstet mit diesem linguistischen Vorwissen können wir nun versuchen, die beiden Strukturen, bei denen der "Sicherheitsabstand" zwischen den beiden Satztypen am geringsten ist, mit Hilfe der Abfragemöglichkeiten von COSMAS II automatisch aus einem digitalen Korpus zu extrahieren. Die so erhobenen Belegmengen werden im Anschluss daran einer philologischen Feinanalyse unterzogen mit dem Ziel, die hier herrschenden, ziemlich subtilen grammatischen und lexikalischen Regularitäten zumindest ansatzweise aufzudecken.

\footnotetext{
10 Vgl. dazu etwa Bist du [aber] gross geworden! vs. *Bist du [aber] vierzehnjährig geworden! (Näf 1996: 141). Die inhaltliche Leistung der Exklamativstruktur kann man so umschreiben: Mit Nachdruck sagen, dass bzw. wie sehr etwas der Fall ist - gegen oder über die Erwartung hinaus.

${ }^{11}$ Nicht möglich sind an dieser Strukturstelle offenbar das Personalpronomen der dritten Person und das dieserDemonstrativpronomen: [Mensch,] Hat DER Hunger!/*Hat ER Hunger!/*Hat DIESer Hunger!. Das Personalpronomen der 3. Person Plural wird in dieser Verwendung wohl automatisch als Höflichkeitsform interpretiert (Haben Sie Hunger! vs. *Haben sie Hunger!).
} 
Unser "Angriffshebel" ist hier also ein doppelter. Zum einen gehen wir von satzeinleitenden Wort-Digrammen wie in (7) aus (prinzipiell kommen die Personalformen aller Tempora im Indikativ und Konjunktiv II von sein und haben in Frage):
Bin/War ich ...
- Habe/Hatte ich ...
Bist/Warst du ...
- Hast/Hattest du ...
Sind/Waren wir ...
- Haben/Hatten wir ...
Ist/War/Wäre der-die-das ...
- $\quad$ Hat/Hatte/Hätte der-die-das ...
Sind/Waren/Wären die-Sie ... $\quad$ - $\quad$ Haben/Hatten/Hätten die-Sie ...

Zum andern erfolgt ein Zugriff auf die Satzschlusszeichen. Durch eine solche Recherche lassen sich alle Sätze mit einem Punkt, Fragezeichen oder Ausrufezeichen am Ende aus dem Korpus extrahieren. ${ }^{12}$ Der relative Anteil der drei Satzzeichen im zta-Korpus geht aus Tab. 4 hervor.

\begin{tabular}{|l|r|r|}
\hline & Treffer absolut & Treffer prozentual \\
\hline Punktsätze & $3{ }^{\prime} 341^{\prime} 911$ & $95.7 \%$ \\
\hline Fragezeichensätze & $124 ' 039$ & $3.6 \%$ \\
\hline Ausrufezeichensätze & $25 ' 736$ & $0.7 \%$ \\
\hline Total & $3^{\prime} 491^{\prime} 686$ & $100 \%$ \\
\hline
\end{tabular}

Tab. 4: Anteil der drei Satzzeichen im zta-Korpus

Da jedoch zwischen Satzschlusszeichen und Satzart keine Eins-zu-Eins-Beziehung herrscht (vgl. Tab. 5), sind die so gewonnenen Belegmengen natürlich nicht ohne weiteres mit einer bestimmten Satzart gleichzusetzen. Immerhin wird man aber behaupten können, dass ein Grossteil der Punktsätze Deklarativsätze und der Fragezeichensätze Interrogativsätze sind.

\begin{tabular}{|lll|}
\hline Punktsätze & $\neq$ & Deklarativsätze \\
Fragezeichensätze & $\neq$ & Interrogativsätze \\
Ausrufezeichensätze & $\neq$ & Exklamativsätze \\
\hline
\end{tabular}

Tab. 5: Keine Eins-zu-eins-Beziehung zwischen Satzschlusszeichen und Satzart

Durch eine Kombinationssuche, beispielsweise mit dem Suchbefehl $<$ Bin ich $>$ UND $<$ Satzschlusszeichen X>, erhalten wir nun als "Rohmaterial" alle Punktsätze, Fragezeichensätze bzw. Ausrufezeichensätze, die jeweils mit den beiden Eingangswörtern beginnen.

Das Globalergebnis einer solchen kombinierten Suchanfrage - demonstriert am Teilkorpus zta - mit den drei exemplarisch herausgegriffenen Satzanfängen Bin ich, War das und Haben wir ist in Tabelle 6 zusammengefasst.

12 Alle Satzzeichen sind prinzipiell mehrdeutig. Der Punkt kann neben der Funktion Satzschlusszeichen beispielsweise auch Abkürzungspunkt oder Ordinalzahlenpunkt sein (vgl. Lemnitzer/Zinsmeister 2006: 65). 
Syntaktische Explorationen anhand von COSMAS II

\begin{tabular}{|l|r|r|r|r|}
\hline & Punktsätze & Fragezeichensätze & Ausrufezeichensätze & Total \\
\hline Bin ich ... & 15 & 74 & 3 & 92 \\
\hline War das ... & 35 & 191 & 7 & 233 \\
\hline Haben wir ... & 18 & 116 & 5 & 139 \\
\hline Total & 68 & 381 & 15 & 464 \\
\hline
\end{tabular}

Tabelle 6: Verteilung der Belege: satzeinleitendes Digramm x Satzschlusszeichen (zta-Korpus)

Generell kann man zur Interpretation der Tabellen 4 und 6 zunächst einmal zweierlei festhalten. Im zta-Korpus als Ganzem gibt es insgesamt 27mal mehr Punktsätze als Fragezeichensätze und 130mal mehr Punktsätze als Ausrufezeichensätze. Bei den drei ausgewählten satzeinleitenden Digrammen überwiegen dagegen bei weitem die Interrogativsätze, während die Ausrufezeichensätze auch hier das Schlusslicht bilden. Darauf soll nun im folgenden näher eingegangen werden.

\subsubsection{Bin ich-Sätze}

Zunächst nun zu den Bin ich-Sätzen. Wie aus Tab. 6 ersichtlich, sind von den insgesamt 92 Bin ich-Belegsätzen des zta-Korpus 74 Fragezeichensätze, 15 Punktsätze und 3 Ausrufezeichensätze.

Alle 74 Fragezeichensätze erweisen sich bei näherer Überprüfung als Interrogativsätze (mit unterschiedlichen Sprechaktwerten), vgl. etwa die Belege in (8).

(8) (a) Bin ich jetzt dran? (zta 24.10.98)

(b) Bin ich wirklich der erste? (zta 02.09.97)

(c) Bin ich schlichtweg out, oder bin ich einfach zu alt? (zta 29.08.98)

Bei den 15 Punktsätzen handelt es sich überwiegend (6 Belege) um uneingeleitete Nebensätze (Vordersätze) im Rahmen eines komplexen Satzes (Deklarativsatzes), vgl. (9). Bei den übrigen Belegen liegen - abgesehen von 5 Irrläufern bzw. Sonderfällen ${ }^{13}-$ drei Deklarativsätze mit Vorfeldellipse (10) und ein Exklamativsatz (11) vor.

(9) Bin ich zufrieden damit, gebe ich sogleich eine Bestellung auf. (zta 13.09.96)

(10) [Darüber scheinen Sie selbst etwas überrascht zu sein.] Bin ich auch. (zta 02.10.99)

(11) Bin ich froh, gibt es das Sechseläuten. (zta 10.04.00)

Und was schliesslich die 3 Ausrufezeichensätze betrifft: 2 davon sind Exklamativsätze (12) und einer ein uneingeleiteter Nebensatz.

(12) "Bin ich froh, dass es nicht meine Tochter ist! ..." (19.07.99)

Dieses durch eine qualitative Interpretation der Daten gewonnene Ergebnis wird unten in Tabelle 7 nochmals resümiert und gedeutet.

\footnotetext{
${ }^{13}$ Es handelt sich um Interrogativsätze, bei denen vom Suchprogramm wegen des fehlenden Zwischenraums fälschlicherweise der Punkt als Satzschlusszeichen aufgefasst wird. In einem Fall wird foreigner talk nachgeahmt: Roy sagte: "Bin ich grosses Zauberer. Kann ich können Aktienkurs lesen ..." (zta 06.08.99).
} 


\subsubsection{War das-Sätze}

Bei den 191 Fragezeichensätzen ${ }^{14}$ (vgl. Tab. 6) handelt es sich samt und sonders um wInterrogativsätze, vgl. (13a-e)

(13) (a) War das eine gute Idee? (zta 05.01.00)

(b) War das früher anders? (zta 28.04.97)

(c) War das wirklich nötig? (zta 24.12.96)

(d) War das nicht ein fataler Fehler? (zta 12.02.00)

(e) War das für Sie nie ein Widerspruch? (zta 15.10.99)

Bei einem kleinen Teil der Interrogativsätze liegt im übrigen nicht das Demonstrativpronomen das vor, sondern der homographe Definitartikel (War das Leben früher einfach spannender als heute? zta 21.11.98), eine Differenzierung, welche in einem nicht getaggten Korpus ebenfalls nur per Handanalyse möglich ist.

Demgegenüber dominieren bei den 35 Punktsätzen mit 23 Belegen (66 \%) ganz klar die uneingeleiteten Nebensätze, und zwar überraschenderweise 19 mal in einem Satzgefüge mit adversativem (15a) und bloss $4 \mathrm{mal}$ in einem solchen mit konditionalem (15b) Bedeutungsverhältnis. ${ }^{15} \mathrm{Sie}$ werden gefolgt von den Exklamativsätzen (6 Belege, vgl. (16a/b) und den Interrogativsätzen (4 Belege), ${ }^{16}$ neben zwei Deklarativsätzen ohne Vorfeld (vgl. "Sonstiges" unten in Tab. 7).

(15) (a) War das Snowboarden anfangs auf manchen Pisten noch verboten [...], empfängt man die Carver mit offenen Armen. (zta 18.12.96)

(b) War das Risiko nicht voraussehbar, muss er den vorausbezahlten Preis zurückzahlen. (zta 25-03.99)

(16) (a) War das ein Stress. (zta 06.07.98)

(b) War das ein Spektakel, als Winterthur vor fünfeinhalb Jahren sein Stadtmarketing lancierte. (zta 02.11.99)

Einfach ist der Befund bei den Ausrufezeichensätzen: 6 von 7 sind Exklamativsätze wie in $(17 \mathrm{a} / \mathrm{b})$, einer ein Interrogativsatz.

(17) (a) War das ein Fest! (zta 12.09.98)

(b) War das eine Aufregung am nächsten Tage! (21.10.96)

Bei den War das-Sätze im zta-Korpus handelt es sich also zum grössten Teil (196 auf 233 oder $80 \%$ ) um Interrogativsätze, bloss 5\% sind Exklamativsätze (vgl. Tab. 7 unten). Wir wollen hier noch kurz aufzeigen, wie man durch Anfragezuspitzung - bei gleichzeitiger Erweiterung des Korpus - die Auftretensbedingungen für die eine oder die andere Struktur noch weiter präzisieren kann. Eine feinere Analyse der zta-Belege führt zum Ergebnis, dass

\footnotetext{
${ }^{14}$ Bei einem von den total 192 angezeigten Treffern handelt es sich um einen Irrläufer, den wir ausgeschieden haben.

15 Die keineswegs marginale adversative Verwendung ist der Duden-Grammatik offenbar entgangen ( ${ }^{7} 2005$ : 1793).

${ }^{16}$ Auch hier liegen Sonderbedingungen infolge fehlerhafter Interpunktion oder Schwierigkeiten des Suchprogramms mit Satzzeichenhäufungen vor.
} 
bei den Exklamativsätzen das Prädikativ das Merkmal [+ indefinite Determination] aufweist (War DAS ein fataler Fehler! vs. *War DAS der fatale Fehler!), eine Restriktion, die für den Interrogativsatz nicht besteht. Der tiefere Grund dafür ist offenbar, dass es bei der Exklamation nie um Gleichsetzung, sondern stets um Klassifizierung geht. War das eine Aufregung! bedeutet, dass es sich im Vergleich zu andern stattgehabten oder bloss potentiellen Aufregungen um eine (in irgendeiner Hinsicht) besonders ausgeprägte, extreme Aufregung handelt. Mit Hilfe von Exklamativsätzen wird bekanntlich eine Normabweichung bezüglich einer Normalerwartung ausgedrückt; ${ }^{17}$ dies ist der Grund, warum ein Satz wie ?War das eine ganz normale/banale Aufregung! kaum sprachgerecht erscheint.

Ausgehend von dieser Hypothese haben wir nun im (rund 20 mal grösseren) COSMAS public-Korpus nach allen Sätzen mit dem Trigramm War das ein/eine am Satzanfang (also mit hinzugefügtem Indefinitartikel) gesucht. Die Erwartung war dabei, dass bei dieser spezifischeren Struktur der Anteil der Exklamativsätze stark steigen würde. Und dies ist tatsächlich auch der Fall: Von den total 412 einschlägigen Belegen erweisen sich nämlich zwei Drittel (267 oder $65 \%$ ) als Exklamativsätze, gegenüber einem Drittel Interrogativsätzen (142 oder $34 \%) .{ }^{18}$ Das heisst: durch einen solchen spezifischeren Suchzugriff haben sich nun die Mehrheitsverhältnisse umgedreht.

$\mathrm{Zu}$ den häufigsten an dieser Strukturstelle im public-Korpus auftretenden Nomen gehören Fest/-fest (Familien-, Geburtstagsfest) (15 Belege), Freude (12 Belege), Krimi (11), Tag (10), Überraschung (8), Hallo (8), Aufregung (6), Wochenende (6) Jubel (5), Spektakel (4) und Schock (4). Um kein Missverständnis aufkommen zu lassen: Im Prinzip kann an dieser Strukturstelle jedes Nomen sowohl in einem Interrogativ- als auch in einem Exklamativsatz als Prädikativ vorkommen. Für Überraschung, Enttäuschung, Schock und Spiel finden sich denn auch im public-Korpus Belege sowohl für die eine als auch die andere Satzart, z.B. (18) vs. (19)

(18) Sie haben Ihren Chinaaufenthalt von langer Hand vorbereitet und konnten nicht einmal ein Jahr in Peking bleiben.] War das eine Enttäuschung? (St. Galler Tagblatt 17.07.99)

(19) [Am Sonntag haben wir die Grün 98 besucht.] War das eine Enttäuschung. (St. Galler Tagblatt 14.04.98)

Trotz dieser prinzipiellen Offenheit tritt aber bei den Exklamativsätzen an dieser Strukturstelle de facto bloss eine relativ kleine Zahl von - semantisch recht klar eingrenzbaren Nomina auf, die sich um folgende Archilexeme gruppieren:

- FREUDE (Freude, Jubel, Hallo, Triumph, Euphorie, Wonne, u.ä.)

- FEST (Fest, Spass, Spektakel, Krimi, Finish, u.ä.)

- ÜBERRASCHUNG (Überraschung, Aufregung, Nervenkitzel, Stress, Frust, Schreck, u.ä.)

- MENSCHENMENGE (Auflauf, Aufruhr, Gedränge, Getümmel, u. ä.)

\footnotetext{
17 Dieser Argumentationsstrang wird entwickelt bei Näf (2005: 248ff.).

18 Bei insgesamt bloss 3 Belegen handelt es sich um Nebensätze.
} 


\section{- ZEITAUSDRUCK (Tag, Nacht, Abend, Woche, Wochenende, Sonntag, u. ä.)}

Aber wir sind damit noch nicht am Ende: Dank dem Belegmaterial aus dem public-Korpus kann man die prototypische Verb-Erst-Exklamativstruktur bezüglich ihrer syntaktischen und semantischen Affinitäten noch weiter einkreisen. An sich kaum speziell erwähnenswert wäre die Tatsache, dass beim prädikativen Nomen ein qualifizierendes Adjektiv stehen kann:

(20) War das ein schöner/toller/herrlicher/aufregender/verrückter usw. X (X = Tag/Abend/Sonntag usw.)

Von Interesse ist nun aber, dass nur gerade $12 \%$ aller einschlägigen Strukturen ein solches Adjektiv aufweisen. Dass ein solches typischerweise gerade nicht steht, hat sicher damit zu tun, dass es pleonastisch wirkt, dies deshalb weil ja schon die grammatische Struktur als solche die Normabweichung (mit dem positiven Wert als default value) transportiert. Obligatorisch scheint das Adjektiv hingegen dann zu sein, wenn es sich um eine extreme negative Bewertung handelt, wie z.B. in

(21) War das ein rabenschwarzer Tag für Niederösterreichs Eishockey. (Neue Kronen-Zeitung 23.01.95)

Zum Ausdruck des besonders ausgeprägten Charakters kommen im übrigen gelegentlich auch augmentative Halbpräfixe zum Einsatz, z.B. War das ein Riesenspass/Supermatch/Klassespiel u. ä.

Zwei letzte Präzisierungen zu den Verb-Erst-Exklamativsätzen. In knapp $10 \%$ der hier zu Debatte stehenden Struktur wird diese gefolgt von einem temporalen als-Satz, wie etwa in (22) oder oben (16b).

(22) War das eine Freude, als die Kinder später die Nester entdeckten! (Mannheimer Morgen 19.04.03)

Und schliesslich noch ein höchst bemerkenswerter Negativbefund: In keinem einzigen der 267 Verb-Erst-Exklamativsätze tritt eine Modalpartikel auf! Dieser Befund steht in eklatantem Widerspruch zu den Aussagen der bisherigen Forschung, die auch für diesen Subtyp das Auftreten von Modalpartikeln als konstitutives Merkmal postuliert hat. ${ }^{19}$

\subsubsection{Haben wir-Sätze}

Wir können uns hier kurz fassen. Insgesamt bietet das zta-Korpus 139 Belege. Bei den 116 Fragezeichensätzen (vgl. Tab. 6 oben) handelt es sich samt und sonders um Interrogativsätze, z. B. (23). Unter den 18 Punktsätzen finden sich 9 Nebensätze, z.B. (24), und 6 Deklarativsätze mit Vorfeld-Ellipse, und unter den 5 Ausrufezeichensätzen 3 Imperativsätze (sog. adhortativer Imperativ), vgl. (25).

\footnotetext{
${ }^{19}$ Gemäss Altmann (1993: 1026) ist das Auftreten einer Modalpartikel hier "vermutlich obligatorisch"; vgl. dazu Näf (1996: 144ff.).
} 
(23) Haben wir nichts gelernt aus der Vergangenheit? (zta 07.05.99)

(24) "Haben wir zuviel Geld, werben wir mit den Hypothekarzinsen, haben wir zuwenig [sic], inserieren wir unsere Sparzinsen", sagt Verwalter Müller. (zta 06.05.96)

(25) "Haben wir den Mut und packen wir es an!" (zta 13.07.96)

Das heisst mit andern Worten, dass sich hier kein einziger Exklamativsatz findet. Dass es sich dabei aber nur um eine zufällig Lücke handelt, lässt sich durch eine Korpuserweiterung schnell nachweisen. Unter den 42 Treffern für <Haben wir> UND <Ausrufezeichen> im public-Gesamtkorpus finden sich insgesamt 6 Exklamativsätze, darunter (26a) und (26b)

(26) (a) Haben wir gelacht! (Die Presse 15.07.00)

(b) Haben wir uns gelangweilt! (Frankfurter Rundschau 15.01.99)

\subsubsection{Zusammenfassung}

Während die automatisch erhobene Tabelle 6 die Verteilung der Belege auf die Satzschlusszeichen zeigt, bietet Tabelle 7 eine von Hand "bereinigte" Auflistung derselben Datenmenge nach den Satzarten.

\begin{tabular}{|l|r|r|r|r|r|}
\hline & $\begin{array}{r}\text { Interrogativ- } \\
\text { satz }\end{array}$ & $\begin{array}{r}\text { Exklamativ- } \\
\text { satz }\end{array}$ & $\begin{array}{r}\text { Uneinge- } \\
\text { leiteter NS }\end{array}$ & Sonstiges & Total \\
\hline Bin ich ... & 74 & 3 & 7 & 8 & 92 \\
\hline War das ... & 196 & 12 & 23 & 2 & 233 \\
\hline Haben wir ... & 119 & 0 & 9 & 11 & 139 \\
\hline Total & $\mathbf{3 8 9}$ & $\mathbf{1 5}$ & 39 & 21 & 464 \\
& $84 \%$ & $3.2 \%$ & $8.4 \%$ & $4.5 \%$ & $100 \%$ \\
\hline
\end{tabular}

Tab. 7: Verteilung der satzeinleitenden Digramme auf die Satzarten (zta-Korpus)

Diese Handauswertung der Verb-Erst-Sätze zeigt zum einen das klare dominieren der Interrogativsätze (84 \%), zum andern deren relativ engen Bezug zum Satzschlusszeichen Fragezeichen. Das bedeutet: Mit einer Suche nach den Fragezeichensätzen kann man mit einer relativ guten Annäherung die Menge der Interrogativsätze erfassen (vgl. Tab. 6 und 7). Die insgesamt 381 Fragezeichensätzen erweisen sich ausnahmslos alle als Interrogativsätze; zu diesen muss man noch 8 weitere Interrogativsätze mit einem andern Satzschlusszeichen hinzuzählen.

Weniger eindeutig fällt ein entsprechender Konnex bei den andern Satzarten aus. Von den total 15 Exklamativsätzen enden 8 mit einem Ausrufezeichen und 7 mit einem Punkt. Das Ausrufezeichen stellt somit weder ein notwendiges noch ein hinreichendes Kriterium für die Satzart Exklamativsatz dar.

Von grossem Interesse ist im übrigen auch hier ein abschliessender Blick auf die Verwendung von Modalpartikeln in den untersuchten Belegen. Wenn wir das nicht negierende nicht und das Satzadverb wirklich zu diesen dazurechnen, findet sich in jedem fünften Interrogativsatz (83 auf 389 oder $21 \%$ ) eine Modalpartikel, und zwar in folgender Frequenz: nicht (42 Belege), wirklich (17), denn (13), überhaupt (5), etwa (4), wohl (2), vgl. etwa die Belege (27) und 
(28). Von den 15 Exklamativsatzbelegen weist dagegen nur ein einziger eine Modalpartikel auf, und zwar doch (29). Wenn man nun darüber hinaus berücksichtigt, dass kein einziger der 267 War das-Exklamativsätze des public-Gesamtkorpus eine solche enthält (s. oben 4.1.2), wird man die diesbezügliche jahrzehntelange Forschungskontroverse - zumindest was das geschriebene Deutsche betrifft - mit der folgenden Feststellung abschliessend so resümieren dürfen: Der prototypische Verb-Erst-Exklamativsatz weist keine Modalpartikel auf.

(27) Haben wir es hier in Zürich nicht viel besser? (zta 17.03.00)

(28) Haben wir denn bisher alles falsch gemacht? (zta 25.03.97)

(29) War das doch ein schönes Lied. (zta 07.02.96)

\subsection{Verb-Zweit-Sätze}

Die Kategorie Verb-Zweit-Satz ist bekanntlich ein Sammelbegriff für eine grössere Zahl von funktional sehr unterschiedlichen Phänomenen, von denen die frequentesten der Deklarativsatz (Ich bin sehr aufgeregt), der w-Interrogativsatz (Warum bist du so aufgeregt?) und der uneingeleitete Verb-Zweit-Nebensatz (Er sagte, er sei sehr aufgeregt) sein dürften. Bei den mit wie und was eingeleiteten Sätzen kann es sich entweder um Interrogativsätze oder um Exklamativsätze handeln. Um die Bedingungen des Auftretens der einen oder anderen Struktur besser in den Griff zu bekommen, soll auch hier exemplarisch auf eine automatische Korpusanalyse zurückgegriffen werden, und zwar mittels einer Kombinationssuche $<$ Wie+Adjektiv $>$ UND $<$ Satzschlusszeichen X>. Beim hier auftretenden wie handelt es sich im übrigen durchs Band um das quantopere-wie ('wie sehr') wie zum Beispiel in Wie habe ich geweint in jenen Tagen (E. Zopfi). ${ }^{20}$

Wie an einem intonatorischen Minimalpaar wie in (30) ersichtlich, stehen den wieInterrogativsätzen auf Seiten des wie-Exklamativsatzes zwei Strukturvarianten (mit Zweitbzw. Endstellung des Finitums) gegenüber:

(30) (a) Wie kompliziert ist das?

(b) Wie kompliziert ist das!/Wie kompliziert das ist!

Neben Adjektiven treten an dieser Strukturstelle auch die Adverbien oft, gerne und lange auf; deren Affinität zu den Adjektiven zeigt sich im übrigen auch darin, dass sie komparierbar sind, weshalb wir sie hier auch unter "ADJ" subsumieren. Nach einer exemplarischen Analyse der Wie oft-Sätze sollen noch einige Vermutungen über die Satzartenpräferenzen anderer häufiger satzeinleitender Digramme formuliert werden.

20 Die terminologische Unterscheidung zwischen quomodo-wie (Wie macht man das?) und quantopere-wie wurde vom Verfasser vor rund zwanzig Jahren in die wissenschaftliche Diskussion eingebracht, vgl. Näf (1987). 


\subsubsection{Wie oft-Sätze}

Ausgangspunkt unserer Analyse bildet die folgende Häufigkeitstabelle für die drei Satzschlusszeichen aus dem zta-Korpus und dem public-Gesamtkorpus:

\begin{tabular}{|l|rr|rr|r|}
\hline Satzschlusszeichen & & zta-Korpus & public-Korpus (exklusiv zta) & public-Korpus total \\
\hline Punktsätze & 43 & $44.3 \%$ & 739 & $47.5 \%$ & 782 \\
\hline Fragezeichensätze & 46 & $47.4 \%$ & 715 & $46.0 \%$ & 761 \\
\hline Ausrufezeichensätze & 8 & $8.2 \%$ & 99 & $6.4 \%$ & 107 \\
\hline Total & 97 & $100 \%$ & 1553 & $100 \%$ & 1650 \\
\hline
\end{tabular}

Tab. 8: Satzschlusszeichen bei den Wie oft-Sätzen (zta und public-Gesamtkorpus)

Aus der Tabelle 8 geht unmittelbar hervor, dass das zta-Teilkorpus eine ganz ähnliche Häufigkeitsverteilung wie das public-Korpus aufweist. Die Durchführung eines t-Tests für unabhängige Stichproben ergibt bei zweiseitiger Fragestellung einen Wert von t $=2.31(\mathrm{df}=4)$ und erreicht damit nicht den kritischen Wert von 2,78 (Signifikanzniveau p=0.05). Die Verteilungsunterschiede zwischen den beiden Stichproben sind somit nicht signifikant. Das ztaKorpus kann deshalb bei unserer Fragestellung für Handanalysen als repräsentative Stichprobe des Gesamtkorpus herangezogen werden.

Zur Satzartenanalyse: Von den 97 Wie oft-Belegen aus dem zta-Korpus erweisen sich von der Nähe besehen 48 als Interrogativsätze, vgl. (31) und (32) und 34 als Exklamativsätze. Was die Satzzeichen betrifft, enden von den letzteren drei Viertel (insgesamt 26) mit einem Punkt, z.B. (33), und bloss 8 mit einem Ausrufezeichen, z.B. (34). Auf die verbleibenden 15 Belege (überwiegend Nebensätze) soll hier nicht näher eingegangen werden. ${ }^{21}$

(31) "Wie oft gehen Sie ins Kino?" (zta 13.08.97)

(32) "Wie oft brauchst du Sex?" (zta 07.03.97)

(33) Wie oft bin ich im Kopf schon abgereist. (zta 05.12.97)

(34) Wir oft wurde mir diese Frage schon gestellt! (zta 15.05.96)

Was die Stellung des finiten Verbs im Exklamativsatz betrifft, weisen ausnahmslos alle 34 Belege Zweitstellung auf! Dieser Befund ist von grösstem Interesse, wenn man sich die immer noch andauernde Forschungskontroverse über diesen Punkt vor Augen hält. ${ }^{22}$

Modalpartikeln treten bei den Interrogativsätzen bloss in insgesamt 3 Belegen auf (2x denn, 1x wohl), und bei den Exklamativsätzen ebenfalls nur in 3 Fällen, wobei es sich dabei stets um doch handelt, vgl. (35). Damit scheinen Modalpartikeln bei den Verb-ZweitExklamativsätzen mit $8 \%$ etwas verbreiteter zu sein als bei den Verb-Erst-Strukturen.

\footnotetext{
${ }^{21}$ Neben "normalen" Nebensätzen treten hier öfter auch verkürzte Nebensätze auf, z. B. Wie oft traf es vor allem die Armen. Im Kontext ist kein Exklamativsatz intendiert, sondern so etwas wie Wie [dies in solchen Fällen] oft [der Fall ist], traf es vor allem die Armen.

${ }^{22}$ Gemäss den Untersuchungen von Näf (1996: 142ff.) überwiegt Zweitstellung, insbesondere bei den periphrastischen Tempora.
} 
(35) Wie oft hat sie doch in den vergangenen zwei Jahren vom Sterben gesprochen. (zta 21.01.97)

In einer vom Verfasser noch "zu vordigitalen Zeiten" von Hand erhobenen Belegsammlung zeichnete sich bei den Wie-Interrogativsätzen und den Wie-Exklamativsätzen eine je unterschiedliche Affinität zu den grammatischen Personen ab. Während sich bei den WieInterrogativsätze eine Präferenz für die Rolle des Angesprochenen (2. Sg./Pl. und Sie-Form) manifestierte, zeigte sich bei den Wie-Exklamativsätzen eine solche für die Sprecherrolle (1. Sg./Pl.). Demgegenüber scheint die 3. Person Sg./Pl. diesbezüglich neutral zu sein. Dies hat natürlich damit zu tun, dass Interrogativsätze - um es in der Terminologie Karl Bühlers auszudrücken - dem "Appell" dienen und sich an einen Gesprächspartner (mit der Bitte um eine Antwort) wenden, die Exklamativsätze aber der "Kundgabe" von eigenen Gedanken und Gefühlen. Prototypische Verwendungsweisen wären also die folgenden (konstruierte Beispielsätze):

Wie oft hast du/habt ihr/haben Sie ihn eingeladen?

Wie oft habe ich/haben wir ihn eingeladen!

Wie oft hat er/haben sie ihn eingeladen?!!

Um diese weitreichende Hypothese mindestens ansatzweise einer Überprüfung zu unterziehen, habe ich für die 1650 Wie oft-Sätze aus dem public-Gesamtkorpus (vgl. Tab. 8) eine automatische Kookkurrenzanalyse durchgeführt. Während sich für die Ausrufezeichensätze (107 Belege) die Zahl der Treffer als zu klein erwies, ist dagegen das Resultat für die Punktsätze (782 Belege) und Fragezeichensätze (761 Belege) sehr aufschlussreich ausgefallen. Bei den Wie-oft-Punktsätzen sind die drei "obersten" - nach absteigendem LLR-Wert angeordneten - Kookkurrenten schon (92 Belege), ich (73) und wir (56), bei den Wie-oftFragezeichensätzen dagegen Sie (116), schon (59), trainierst (6), denn (21) und du (14). Es ist also tatsächlich so, das die Exklamativsätze (diese machen das Gros der Punktsätze aus) mit den Personalpronomen der Sprecherrolle (ich, wir) korrelieren, die Interrogativsätze hingegen mit den Anredepronomen (Sie, $d u$ ). Darüber hinaus wird deutlich, dass das Adverb schon - in beiden Satzarten - überzufällig häufig auftritt (vgl. etwa die obigen Belege (33) und (34)) und dass bei den Interrogativsätzen eine Modalpartikel (denn) unter den affinsten Kookkurrenten figuriert (als weitere Modalpartikel findet sich auf Rang acht noch eigentlich mit 13 Belegen). Alles in allem bestätigt sich somit unsere Vermutung, dass zwischen Satzart und grammatischer Person (markiert durch das Subjektspronomen und das mit diesem kongruierende Verb) eine überzufällige Affinität besteht.

\subsubsection{Wie+ADJ-Sätze}

Es soll hier noch ein Blick auf andere wichtige Wie+ADJ-Sätze geworfen werden. Wir müssen uns hier aber auf die Formulierung von Vermutungen beschränken angesichts der Tatsache, dass der Zeitaufwand für eine umfassende Aufdeckung der Affinitäten zwischen Adjektiv und Satzart ziemlich gross wäre. 
$\mathrm{Zu}$ den häufigsten und aufschlussreichsten satzeinleitenden Digrammen gehören die folgenden (in Klammern deren Frequenz im public-Korpus):23

\begin{tabular}{|c|c|c|}
\hline Wie lange (5361) & Wie kurz (67) & Wie lustig (28) \\
\hline Wie hoch (2434) & Wie spannend $(65)$ & Wie jung (25) \\
\hline Wie gut (2122) & Wie herrlich (64) & Wie unangenehm (24) \\
\hline Wie oft (1734) & Wie wunderbar (55) & Wie unsicher (23) \\
\hline Wie stark (1364) & Wie selten (55) & Wie furchtbar (21) \\
\hline Wie schön (966) & Wie laut (53) & Wie billig (20) \\
\hline Wie schnell (960) & Wie angenehm (51) & Wie toll (18) \\
\hline Wie gross (673) & Wie kompliziert (47) & Wie ruhig (18) \\
\hline Wie wenig (401) & Wie froh (45) & Wie grausam (18) \\
\hline Wie alt (400) & Wie langweilig (45) & Wie langsam (13) \\
\hline Wie teuer (328) & Wie dumm (40) & Wie dick (13) \\
\hline Wie gern(e) (187) & Wie schrecklich (39) & Wie überrascht (11) \\
\hline Wie sicher (174) & Wie nett (37) & Wie enttäuscht (11) \\
\hline Wie einfach (144) & Wie schwach (36) & Wie erleichtert (9) \\
\hline Wie schlecht (120) & Wie dünn (34) & Wie müde (9) \\
\hline Wie schlimm (95) & Wie traurig (30) & Wie niedrig (9) \\
\hline Wie klein (79) & Wie flexibel (30) & \\
\hline Wie glücklich (73) & Wie interessant (28) & \\
\hline
\end{tabular}

Tab. 9: Auswahl von Digrammen mit Wie+Adjektiv (in absteigender Häufigkeit) (public-Korpus)

Auf der Grundlage eines Globaleindrucks bei der Durchsicht der einschlägigen KWIC-Listen sowie weiterer Überlegungen und Testverfahren wollen wir hier abschliessend noch einige Tendenzen und Erklärungsansätze vorbringen, in der Absicht, darüber eine wissenschaftliche Diskussion in Gang zu setzen. Wir beschränken uns im Folgenden bewusst auf die Dichotomie Interrogativsatz vs. Exklamativsatz. Das heisst zugleich, dass wir hier alle andern Belege, insbesondere die Nebensätze, beiseite lassen. Diese sind indes keineswegs eine Quantité négligeable, sondern machen im Gegenteil bei einzelnen Digrammen bis zur Hälfte und mehr aller Belege aus. Nur nebenbei sei hier angemerkt, dass man nach unserer Auffassung auch bei den wie-ADJ-Nebensätzen zwischen indirekten Interrogativsätzen (transformierbar in einen Interrogativsatz, z.B. (36)) und indirekten Exklamativsätzen (transformierbar in einen Exklamativsatz, z.B. (37)) unterscheiden muss, eine Differenzierung, die jedoch von den gängigen Grammatiken des Deutschen in der Regel nicht vorgenommen wird. ${ }^{24}$
(36) Ich möchte wissen, wie oft du ins Kino gehst.
$(\rightarrow$ Wie oft gehst du ins Kino?)
(37) Ich bin erstaunt, wie unflexibel der ist.
$(\rightarrow$ Wie unflexibel der ist!)

\footnotetext{
${ }^{23}$ Ausgeklammert wurde hier der (infolge von Schreibschwankungen kompliziertere) Fall von Wie viel, Wie viele, Wieviel.

${ }^{24}$ Vgl. dazu Näf (1992: 55ff.). Die neueste Auflage der Duden-Grammatik ('2005: 1052) spricht nun erstmals auch von Ausrufenebensätzen oder Exklamativnebensätzen.
} 
Wie oben in 4.2.1 festgehalten, kommen bei satzeinleitendem Wie oft die beiden Satzarten etwa ähnlich häufig vor (48 Interrogativsätze vs. 34 Exklamativsätze). Auch bei den Wie+ADJ-Sätzen gilt nun zwar, dass bei jedem Adjektiv im Prinzip sowohl ein Interrogativsatz als auch ein Exklamativsatz auftreten kann. Doch lehrt schon ein flüchtiger Blick auf den Gesamtbefund, dass hier deutliche Distributionspräferenzen herrschen. Zwei davon sollen nun versuchsweise noch etwas näher umrissen werden.

Zur ersten Tendenz. Eine deutliche Affinität zu den Exklamativsätzen zeigen jene Adjektive, die eine stark ausgeprägte, ja "extreme" Eigenbedeutung (auf dem negativen oder positiven Pol) aufweisen und starke Gefühle, Werturteile oder Charakterisierungen zum Ausdruck bringen, wie zum Beispiel in den folgenden (aufgrund von entsprechenden Belegen vereinfachten) Serien:

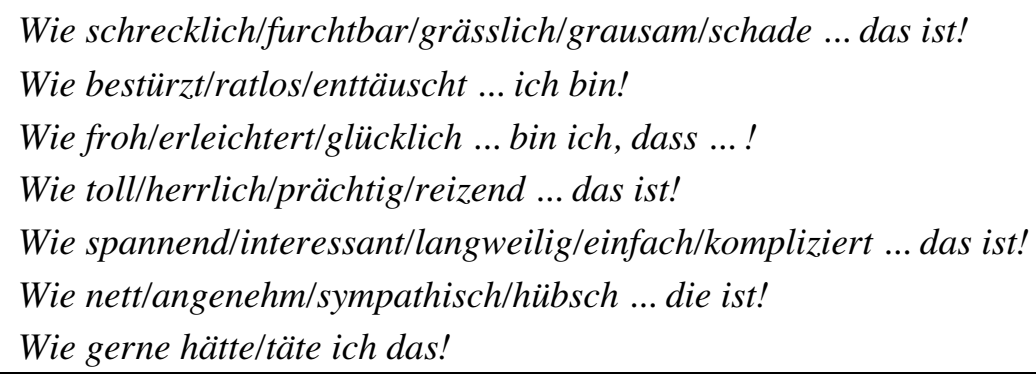

Tab. 10: Adjektive mit starker Affinität zu den Exklamativsätzen

Typische Belege für Exklamativsätze mit diesen Adjektiven sehen so aus:

(38) Wie grausam das Schicksal sein kann! (zta 27.09.96)

(39) "Wie glücklich könnte ein Mann mit seiner Frau leben, wenn er sie nie kennengelernt hätte." (Neue Kronen-Zeitung 16.04.98)

(40) Wie herrlich muss das Leben eines Single in Manhattan sein! (Salzburger Nachrichten 29.03.93)

(41) Wie spannend ist doch eine Stadt, in der das Neue neben und nicht an Stelle des Alten steht! [sic] (zta 28.08.99)

(42) Wie einfach war doch das Sparen früher! (St. Galler Tagblatt 02.07.99)

(43) Wie nett sie mich auf den Arm nahm. (Mannheimer Morgen 29.09.01)

(44) "Wie gerne wäre ich ein grosser Held, der Ungeheuer besiegt und Prinzessinnen rettet!..." (Mannheimer Morgen 19.10.02)

Als Erklärung für das Dominieren der Exklamativsätze können wir uns zurechtlegen, dass diese Satzart ein geeignetes Vehikel für die Prädizierung stark qualifizierender (Selbst)einschätzungen und Beurteilungen ist. Ein Satz wie Wie schrecklich das war! stellt ja im Grunde nichts anderes als eine - emotional allerdings stark aufgeladene - Affirmation dar (paraphrasierbar durch Das war sehr schrecklich). Eine wie-Frage jedoch, welche hier nach dem Grad des Schreckens (oder der Bestürzung, der "Herrlichkeit" usw.) fragen würde (auf einer Skala von "überhaupt nicht" bis zu "sehr stark"), wäre angesichts der Absolutheit der Aussage nicht angebracht und wirkt deshalb auch kaum sprachüblich: 
(46) ?Wie bestürzt bist du jetzt?

(47) ?Wie herrlich schmeckt dir das Bauernbrot?

Um beim Beispiel (47) zu bleiben: Man würde sich bei einem Gesprächspartner mit einer Formulierung wie Wie schmeckt dir das Bauernbrot? erkundigen, ohne ihm dabei wie in dem zu Demonstrationszwecken konstruierten Satz (47) bereits ein qualifizierendes Adjektiv - und schon gar nicht ein derart "extremes" - quasi als Antwort in den Mund zu legen.

Eine Bestätigung für diese Sicht der Dinge liefern solche Adjektive, die einen extremen, kaum mehr steigerbaren Ausprägungsgrad bereits in sich enthalten. Denn während man bei Adjektiven wie interessant, kompliziert oder glücklich noch Abstufungen vornehmen kann (z. B. mit Hilfe von graduierenden Partikeln wie ziemlich, sehr), trifft das für Lexeme wie die folgenden nicht mehr zu: Wie wunderbar, Wie zauberhaft, Wie reizend, Wie phantastisch, Wie entzückend, Wie abscheulich, Wie scheusslich, Wie ekelhaft, usw. Diese können denn auch normalerweise nur in Exklamativsätzen (und nicht in Interrogativsätzen) auftreten.

(48) Wie wunderbar der erste Bissen schmecken muss! (Neue Kronen-Zeitung 06.04.96)

(49) Wie widerlich sieht es doch vielerorts aus; auch Zigarettenreste tragen dazu bei. (St. Galler Tagblatt 19.08.97)

Man vergleiche dazu auch konstruierte Minimalpaare wie etwa die folgenden:
(50) (a) Wie hypersensibel der ist!
vs. ?Wie hypersensibel ist der?
(b) Wie todunglücklich ich damals war!
vs. ?Wie todunglücklich war ich damals?
(c) Wie herzzerreissend der Abschied war!
vs. ?Wie herzzerreissend war der Abschied?

Zur zweiten Tendenz. Bei den quantifizierenden Dimensionsadjektiven (lang, hoch, oft, alt, teuer, usw.), deren präzise Bedeutung sich nur im Hinblick auf eine Norm-Grösse bestimmen lässt, ${ }^{26}$ aber auch bei gewissen qualitativ abstufenden Adjektiven (gut, gefährlich, zufrieden, flexibel, usw.) ist die Opposition zwischen den beiden Antonymen zugunsten des "positiven" Pols neutralisiert. ${ }^{27}$ Wie allgemein bekannt bedeutet in einer Nominalgruppe wie ein zwei Tage altes Baby das Adjektiv alt nicht 'bejahrt', sondern ist eine "reine Altersangabe". Diese Neutralisierung der Bedeutung betrifft aber nur das Vorkommen dieser Adjektive im WieInterrogativsatz, ${ }^{28}$ nicht jedoch im Wie-Exklamativsatz. Dies hat nun zwei verschiedene, aber miteinander in engem Zusammenhang stehende Konsequenzen: Zum einen ist bei den Wie+ADJ-Digrammen das Adjektiv, das die Dimension als solche vertritt (z.B. hoch), sehr viel häufiger belegt als dessen Antonym (z. B. niedrig, vgl. dazu Tab. 11, mit den Frequenzen

\footnotetext{
25 Eine gewisse Ausnahme bildet hier das Digramm Wie schlimm, bei dem Interrogativsätze völlig geläufig sind: Wie schlimm steht es um Österreichs Finanzen? (Vorarlberger Nachrichten 25.02.00).

${ }^{26}$ Das Adjektiv klein bedeutet (in Zentimetern ausgedrückt) nicht dasselbe, je nachdem ob man von einem Haus oder einem Chip spricht.

27 "Positiv" ist bloss ein behelfsmässiger Terminus, der zwar für Paare wie gut-schlecht passt, aber natürlich weniger für solche wie alt-jung.

${ }^{28}$ Und natürlich auch im Deklarativsatz: Ich bin 40 Jahre alt/?jung.
} 
im public-Korpus). Grundsätzlich kann das "dimensionsneutrale" Adjektiv aber sowohl im Interrogativ- als auch im Exklamativsatz auftreten.

Im Gegensatz dazu kommen die jeweiligen Antonyme niedrig, kurz, selten, jung, schlecht, unzufrieden usw. kaum in Wie-Interrogativsätzen vor, sondern finden - abgesehen von den Nebensätzen - fast nur in Wie-Exklamativsätzen Verwendung.

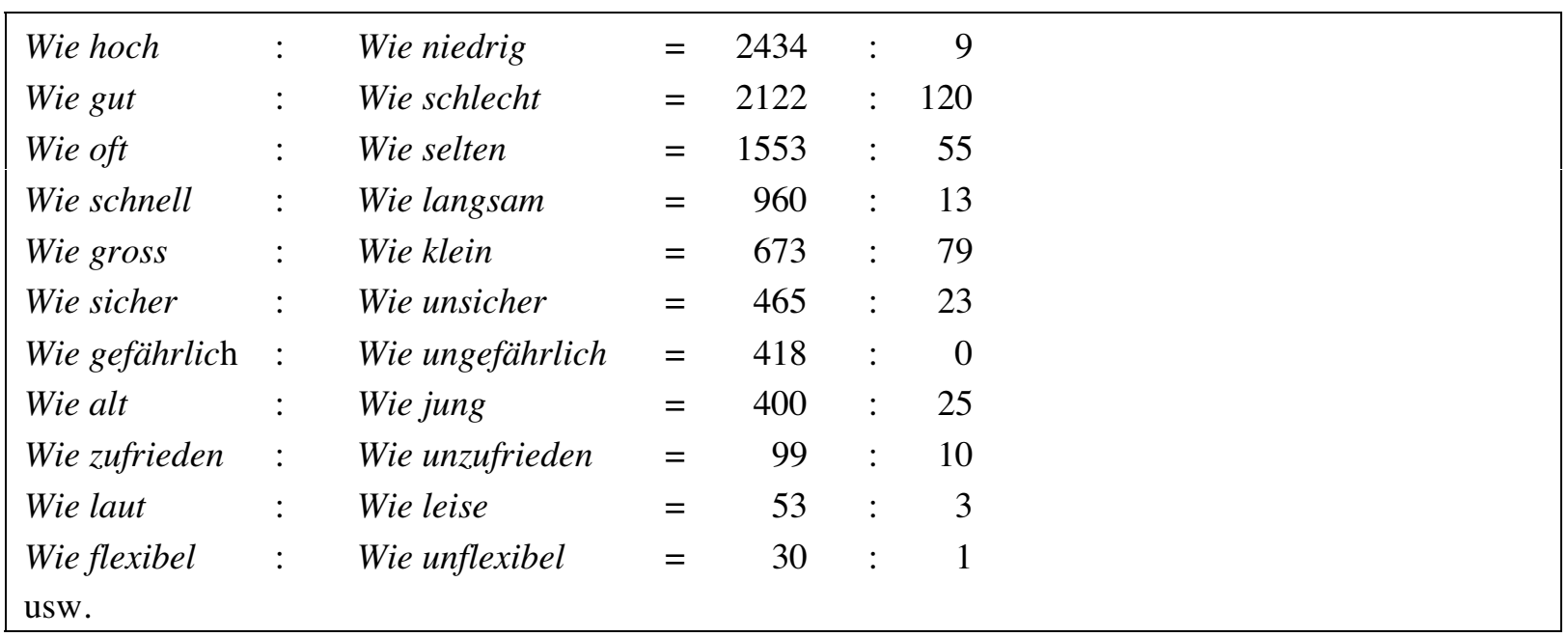

Tab. 11: Auftretenshäufigkeit der Antonyme im public-Gesamtkorpus

Interrogativsätze werden also normalerweise wie folgt formuliert (in Anlehnung an reale Belege teilweise vereinfachte Beispiele):

(51) Wie hoch/?niedrig ist Ihr Einkommen?

(52) Wie gut/?schlecht kannst du Ski fahren?

(53) Wie oft/? selten gehst du ins Kino?

(54) Wie schnell/? langsam darf man auf der Autobahn fahren?

(55) Wie gross/?klein ist deine Wohnung?

(56) Wie flexibel/? unflexibel ist die eigentlich?

(57) Wie gefährlich/? ungefährlich ist Elektrosmog?

Das public-Korpus umfasst Tausende von Interrogativsatz-Belegen mit dimensionsneutral verwendetem Adjektiv, z. B.

(58) Wie gross ist der Einfluss der Medien? (zta 13.03.00)

(59) Wie gut war die alte Zeit? (Die Presse 5.11.91)

(60) "Wie zufrieden sind Sie mit Ihrem Aussehen?" (Die Presse 09.09.00)

(61) Wie sicher sind unsere Arbeitsplätze? (St. Galler Tagblatt 19.06.97)

Bei Vorliegen von bestimmten Spezialbedingungen können aber auch die in den Beispielsätzen (51) bis (57) mit Fragezeichen versehenen Antonyme in Interrogativsätzen auftreten, z. B. wenn man eine jüngere Person - mehr oder weniger scherzhaft - mit Wie jung bist du eigentlich? nach ihrem Alter fragt. Belege für derartige - meist markierte oder in einem ganz 
speziellen Kontext auftretende - Interrogativsätze aus dem public-Korpus sind etwa die folgenden:

(62) Wie klein ist das kleinste Teilchen? (Frankfurter Rundschau 28.02.98)

(63) "Wie schlecht darf Kunst sein". (Die Presse 16.09.94)

(64) Ein sehr seltener Pilz. - Wie selten? (Mannheimer Morgen 07.08.00)

(65) Wie billig darf ein gesundes Osterei sein? (Vorarlberger Nachrichten 26.03.97)

(66) Wie langsam ist eine Strassenbahn? (Die Presse 07.04.94)

(67) Wie dünn ist die Schicht? (Die Presse 13.12.97)

Insgesamt ist es aber so, dass die - vergleichsweise nicht sehr frequenten - Digramme wie Wie klein, Wie jung, Wie billig, Wie unflexibel usw. fast ausschliesslich in Exklamativsätzen auftreten. In diesen herrschen denn auch keinerlei derartige "polare" Beschränkungen, vgl. die jeweils voll akzeptablen (im Anschluss an reale Belege konstruierten) Beispiele (68) und (69)

(68) Wie alt die aussieht! vs. Wie jung die aussieht!

(69) Wie flexibel der ist! vs. Wie unflexibel der ist!

Zum Teil lassen sich auch im Korpus derartige (Beinahe-)Minimalpaare finden:

(70) Wie schnell die Zeit vergeht! (Kleine Zeitung 29.07.99)

(71) Wie langsam die Zeit verrinnt. (St. Galler Tagblatt 29.09.00)

Einschlägige Belege für Exklamativsätze mit negativ-polarem Adjektiv aus dem publicKorpus sind etwa:

(72) Wie niedrig waren unsere Löhne! (Frankfurter Rundschau 17.05.97)

(73) Wie schlecht doch die Welt ist! (Die Presse 25.04.98)

(74) Wie klein die Welt doch ist. (Die Presse 08.09.00)

(75) Wie jung doch Joschka war! (zta 09.06.98)

(76) Wie leise ist es hier, wie heilsam die Luft. (Neue Kronen-Zeitung 30.06.96)

\section{$5 \quad$ Schluss}

Nicht grammatisch annotierte Korpora scheinen auf den ersten Blick für syntaktische Fragestellungen wenig geeignet. Ziel dieses Beitrags ist aber aufzuzeigen, dass sich ein Korpus wie COSMAS II, trotz oder vielleicht sogar wegen der nicht vorhandene Annotierung, ${ }^{29}$ mit Gewinn auch für die Untersuchung hochkomplexer grammatischer Phänomene wie etwa der Satzarten einsetzen lässt. Bei der vorliegenden Untersuchung zur Differenzierung von Satzarten mit Verb-Erst- und Verb-Zweit-Stellung sind wir von linguistischem Vorwissen ausgegangen, das uns durch die Auswertung eines nicht digital verfügbaren und manipulierbaren Korpus zur Verfügung stand. Das COSMAS II-Korpus (Teilkorpus zta und Gesamtkorpus

\footnotetext{
${ }^{29}$ Bekanntlich plädiert ein Teil der Korpuslinguisten dafür, auf eine Annotierung (und damit auf eine gewisse Vorinterpretation) von digitalen Korpora ganz zu verzichten (Lemnitzer/Zinsmeister 2006: 102).
} 
public) diente hier also dazu, durch andere Methoden gewonnene Hypothesen einer Überprüfung zu unterziehen. Wenn man will, kann man hier von einem korpusgestützten Verfahren (im Gegensatz zu einem korpusbasierten) sprechen. ${ }^{30}$ Unsere Arbeitsweise, die sowohl auf quantitative als auch qualitative Prozeduren zurückgreift, beruht auf einer Kombination von automatischer Korpusanalyse und philologischer Handarbeit. Als "Angriffshebel" zur automatischen Belegsuche dienten dabei neben gewissen Funktionswörtern auch Phänomene wie Satzzeichen und Grossschreibung.

Die so mit Hilfe kombinierter Suchanfragen generierten Belegmengen stellen zunächst erst einmal ein Rohmaterial dar. Um nicht mit Belegmaterial überschwemmt zu werden, war es ferner nötig, zum Teil bloss mit einem Teilkorpus zu arbeiten. So haben wir uns auf das ztaKorpus beschränkt, um aus der Menge der automatisch erhobenen Ausrufezeichensätze von Hand die Exklamativsätze herauszufiltern. Wie man durch Anfragezuspitzung, im Verbund mit einer Korpuserweiterung (Rückgriff auf das Gesamtkorpus public), zu noch präziseren und spezifischeren Ergebnissen kommen kann, haben wir exemplarisch anhand des satzeinleitenden Trigramms War das ein(e) aufgezeigt.

Automatisch liessen sich Verb-Erst-Sätze mit Auxiliarverb in Erstposition und Verb-ZweitSätze mit Wie+ADJ aus dem COSMAS-Korpus extrahieren. Danach galt es aber, diese von Hand auf die drei Haupttypen Interrogativsatz, Exklamativsatz und Nebensatz zu verteilen, ein Sortierungsoperation, die gegenwärtig noch nicht automatisch durchgeführt werden kann:
Interrogativsatz
(a) War das eine GUTe Idee?
(b) Wie oft HAT der schon gelogen?
Exklamativsatz
(a) War DAS eine gute Idee!
(b) Wie OFT hat der schon gelogen!
Nebensatz
(a) War das eine gute Idee, (dann ...)
(b) Wie oft der schon gelogen hat, (...)

Gewiss, mit dem hier aufgezeigten Verfahren lassen sich syntaktische Phänomene wie etwa die Interrogativsätze oder die Exklamativsätze nicht direkt anpeilen oder gar automatisch exhaustiv extrahieren. Durch eine gezielte Kombination von Suchbefehlen können wir uns jedoch sozusagen in Sekundenschnelle ein relevantes Belegmaterial von syntaktischen Phänomenen zusammenstellen lassen, das dann für eine anschliessende Handauswertung zur Verfügung steht. Von den Mühen der zeitaufwändigen Belegsuche befreit, kann sich der mit einen digitalen Korpora arbeitende Linguist dann umso intensiver der Auswertung und Interpretation der sprachlichen Fakten widmen, eine "Arbeitsteilung", durch die sich für die künftige grammatische Forschung völlig neue Perspektiven eröffnen.

\footnotetext{
${ }^{30} \mathrm{Vgl}$. dazu die von E. Tognini-Bonelli etablierte Unterscheidung zwischen corpus-based und corpus-driven (Lemnitzer/Zinsmeister 2006: 102).
} 


\section{Literaturangaben}

Albert, Ruth/Koster, Cor J. (2002): Empirie in Linguistik und Sprachlehrforschung. Ein methodologisches Arbeitsbuch. Tübingen.

Altmann, Hans (1993): "Satzmodus". In: Jacobs, J. et al. (eds.). Syntax. Ein internationales Handbuch zeitgenössischer Forschung. 1. Halbband. Berlin: 1006-1029.

Drosdowski, Günther et al. (eds.) $\left({ }^{5} 1995 /{ }^{6} 1998\right)$ : Duden. Grammatik der deutschen Gegenwartssprache. Mannheim.

Dudenredaktion (ed.) ("2005): Duden. Die Grammatik. Unentbehrlich für richtiges Deutsch. Mannheim.

Eroms, Hans-Werner (2000): Syntax der deutschen Sprache. Berlin.

Heid, Ulrich (2005): "Corpusbasierte Gewinnung von Daten zur Interaktion von Lexik und Grammatik: Kollokationen - Distribution - Valenz". In: Lenz, Friedrich/Schierholz, Stefan (eds.) (2005): Corpuslinguistik in Lexik und Grammatik. Tübingen: 97-122.

Helbig, Gerhard/Buscha, Joachim (2001): Deutsche Grammatik. Ein Handbuch für den Ausländerunterricht. Berlin.

Lemnitzer, Lothar/Zinsmeister, Heike (2006): Korpuslinguistik. Eine Einführung. Tübingen.

Lenz, Susanne (2000): Korpuslinguistik. Tübingen. (= Studienbibliographien Sprachwissenschaft 32).

Näf, Anton (1987): "Gibt es Exklamativsätze?". In: Meibauer, Jörg (ed.): Satzmodus zwischen Grammatik und Pragmatik. Tübingen: 140-160.

Näf, Anton (1992): "Herre, wie bin ich mit liebe alsus verwirret! Zu den Exklamativsätzen in Gottfrieds 'Tristan'". ZGL 20: 37-62.

Näf, Anton (1995): "Die Satzarten als Lern- und Reflexionsgegenstand in der Schule". Der Deutschunterricht 47, 4/1995: 51-69.

Näf, Anton (1996): "Die w-Exklamativsätze im Deutschen - zugleich ein Plädoyer für eine Rehabilitierung der Empirie in der Sprachwissenschaft". ZGL 24 (1996): 135-152.

Näf, Anton (2005): "Zum Veralten syntaktischer Strukturen - am Beispiel von Übersetzungen des 'Nibelungenlieds'". In: Corbellari A./Schnyder A. (eds.): Translatio Litterarum ad Penates. Das Mittelalter übersetzen - Traduire le Moyen Âge. Lausanne: 243-269. 